UCRL-JC-122814

PONERINT $-951272-1$

\title{
Self-consistent Core-Edge Nonlinear Transport Simulations with CORSICA 2
}

\author{
PECES E
}

A. Tarditi
R.H. Cohen

G.G. Craddock

J.A. Crotinger

G.D. Porter

T.D. Rognlien

A.I. Shestakov

G.R. Smith
FED O 61936

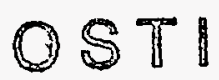

This paper was prepared for submittal to

Plasma Edge Theory Conference

Pacific Grove, CA

December 3-6, 1995

January 17, 1996

This is a preprint of a paperintended for publication in a journal or proceedings. Since changes may be made before publication, this preprint is made available with the understanding that it will not be cited or reproduced without the permission of the author.

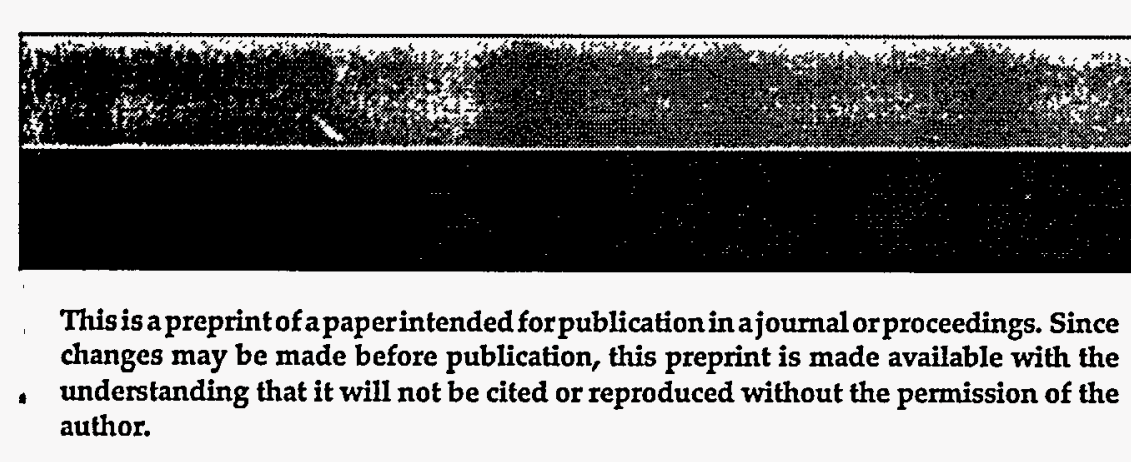


DISCLAIMER

This document was prepared as an account of work sponsored by an agency of the United States Government. Neither the United States Government nor the University of California nor any of their employees, makes any warranty, express or implied, or assumes any legal liability or responsibility for the accuracy, completeness, or usefulness of any information, apparatus, product, or process disclosed, or represents that its use would not infringe privately owned rights. Reference herein to any specific commercial products, process, or service by trade name, trademark, manufacturer, or otherwise, does not necessarily constitute or imply its endorsement, recommendation. or favoring by the United States Government or the University of California. The views and opinions of authors expressed herein do not necessarily state or reflect those of the United States Government or the University of California, and shall not be used for advertising or product endorsement purposes. 


\author{
A. Tarditi, R. H. Cohen, G. G. Craddock J. A. Crotinger, G.D. Porter, T. D. Rognlien, A. I. \\ Shestakov and G. R. Smith
}

Lawrence Livermore National Laboratory, Livermore, CA 94550 (USA)

\begin{abstract}
The coupled core-edge nonlinear transport code CORSICA 2 is introduced and the structure of its iterative coupling algorithm is briefly discussed. Selected application results are reported that reproduce equilibria in DII-D discharges with plasma profiles initialized from the experimental data. Simulations for an L-H transition and for a gas puffing experiment in DIII-D plasmas are presented.
\end{abstract}

\title{
1. INTRODUCTION
}

The modeling of transport in the core and the edge of a magnetically confined fusion plasma can be performed by self-consistently coupling two independent codes [1], [2], [3]. This approach allows to take advantage of the space and time scale separation between these two regions (the edge requires a finer space and time resolution than the core because its smaller radial extension and the rapid losses to the divertor or limiter in the scrape-off layer). Also, the model can be easily implemented by using a one-dimensional (1D, flux-surface averaged) model for the core and a two-dimensional (2D, poloidal and radial) model for the edge.

\section{CORSICA 2 STRUCTURE}

CORSICA 2 is a coupled core-edge nonlinear transport code that includes CORSICA 1, a 1D core transport module coupled with a free-boundary MHD equilibrium module (TEQ) [4], and UEDGE, a 2D axisymmetric tokamak edge model [5]. CORSICA 2 models the transport of energy and particles by following the evolution of the electron and ion temperature and plasma density self-consistently from the center of the tokamak to the scrape off layer region. The first version (CORSICA 2.0) is run with the MHD equilibrium module "frozen" during the time evolution (a limitation that will be soon relaxed). Both the 2D and the 1D transport modules share the same magnetic field configuration computed by solving MHD equilibrium at the beginning of the run.

The core density and temperature profiles are joined to the flux-surface average profiles from the 2D code at a flux surface that is sufficiently inside the magnetic separatrix that

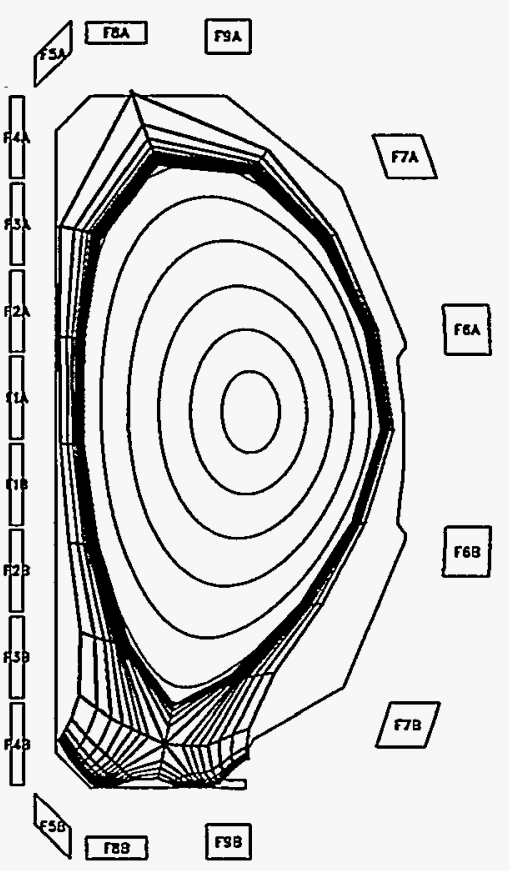

Fig. 1. CORSICA 21D-2D mesh. the edge profiles are approximately constant on that a surface. The resulting 1D-2D mesh is illustrated in Fig. 1.

The core and the edge are advanced together, iterating to obtain a consistent boundary condition. 
Given the vast disparity between the core transport timescale and the edge relaxation timescale, the edge is treated quasi-statically, with $U E D G E$ solving the fluid equations for the steady state. As $U E D G E$ 's asymptotic steady-state solver is much faster than its time dependent solver, this results in a considerable savings in computer time.

In some applications it may be desirable or necessary to follow the coupled system on the edge timescale. Occasionally $U E D G E$ 's asymptotic solver will not converge, possibly because the core has taken too large a timestep, or because the edge solution has reached a bifurcation point. In such cases the code switches the $U E D G E$ solver to the time-dependent mode, advancing with the same $\Delta t$ of the core, and resuming the asymptotic mode as soon as the solution has been completed.

The CORSICA 1 - UEDGE coupling was done at the highest level possible, by considering the codes as modules with specified I/O data stream and by taking advantage of the powerful BASIS interpreter shell [6]. The result is that CORSICA 2 has a very modular structure that only required minor modifications to the core and edge building blocks. The code has shown good convergence properties and some fusion relevant test runs have been performed as DIII-D “demo" cases.

\section{THE ITERATIVE COUPLING SCHEME}

The self-consistency of the simulation is guaranteed, for each coupled variable, by imposing the continuity of the fluxes on both sides of the 1D-2D interface. For a generic variable $u$ this condition is expressed simply as $\Gamma_{u C O R E}=\Gamma_{u E D G E}$, where $\Gamma_{u C O R E}$ is the flux of the variable $u$ across the surface at the 1D-2D interface as computed by the core model and $\Gamma_{u E D G E}$ is the same as computed by the edge code.

$\Gamma_{u C O R E}$ and $\Gamma_{u E D G E}$ are independently defined in both the core and edge codes; the coupling algorithm does not depend on the nature (diffusive, convective) of the fluxes but tries to impose simply a numerical matching for the physical quantity $\Gamma_{u}$ between the $1 \mathrm{D}$ and the $2 \mathrm{D}$ regions.

The self-consistent coupling is performed by means of a Newton predictor-iteration method: the global profile for the variable $u$ will be computed by running both core and edge codes with a Dirichlet boundary condition $u=u_{I}$ imposed on the interface. Starting from an initial guess (usually from the previous time step) the $u_{I}$ that satisfies $f\left(u_{I}\right)=\Gamma_{u C O R E}-\Gamma_{u E D G E}=0$ within a specified tolerance is found through a the Newton scheme on $f\left(u_{I}\right)$.

The coupling is currently performed for three fields (plasma density, electron temperature and ion temperature) so the algorithm is implemented for the multi-variable case (the computation of the Jacobian for the Newton iteration is done numerically by solving the fluid equations after having introduced a small perturbation for each variable on the interface).

Although presently only Dirichlet boundary conditions are applied during the iteration to match the fluxes, the algorithm allows also to impose mixed boundary conditions and iterate to match the interface values on density and temperature.

\section{SIMULATION RESULTS}

As a test of the CORSICA 2 code, an $L-H$ transition in the DIII-D tokamak has been considered. The initial conditions were taken from $L$-mode data for the DIII-D shot \#86586 at $1630 \mathrm{~ms}$. The transport coefficients in the core were chosen such that the initial profiles were in a quasi-steady state and approximately fit the experimental data. The core transport coefficients at the edge were set to the values used in the $U E D G E$ simulation of the shot at the same time. The core coefficients remained fixed throughout the remaining evolution. 
Next the core-edge coupling was enabled. Following a short transient, a new, consistent, steady state was reached.

In order to simulate an $\mathrm{L}-\mathrm{H}$ transition as in the experiment, the fueling and the neutral beam heating were tripled. In the experiment, after $20 \mathrm{~ms}$ of higher beam power, the transition occurs. In the simulation this transition was modelled by decreasing the diffusion coefficient $(D)$ and the thermal conductivities $\left(\chi_{e}\right.$, $\chi_{i}$ ) in the edge (Fig. 2). The new values for $D, \chi_{e}$ and $\chi_{i}$ were chosen from a $U E D G E$ run that fit the $\mathrm{H}$-mode regime data for the same shot at a later time (2550 ms). In this case, the transport co-

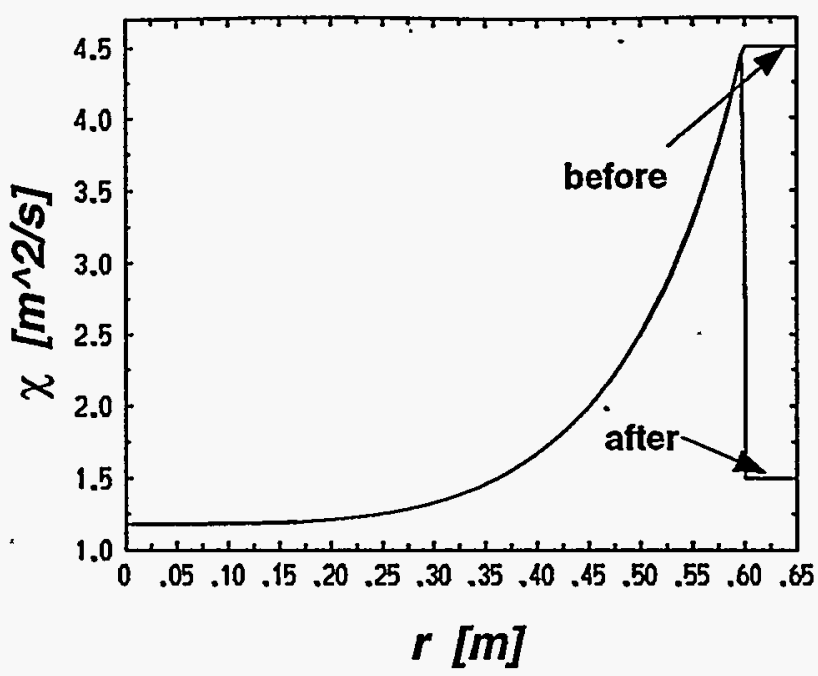

Fig. 2. Dropping $\chi$ In the edge while holding It flxed In the core.

efficients do not exhibit a very strong variation: $D$ drops from 0.4 to 0.3 while the $\chi$ 's were $4.5 \mathrm{~m}^{2} / \mathrm{s}$ and dropped to 1.5 . The decreased edge transport coefficients set up a transport barrier that results in the pedestal-type temperature profiles as shown in Fig. 3.

The code shows sufficient robustness to deal with sharp gradients and was able to achieve a new steady state solution. The steady-state H-mode experimental data were referred to the time $2550 \mathrm{~ms}$. At that time the code had reached more than $90 \%$ of its new regime values. Figure 4 shows a comparison of this $\mathrm{H}$-mode regime for both simulation and experiment (data for the core only).

Another experiment was done to examine the response of the coupled codes to the edge gas puffing. The purpose was to see how the core responds to an edge perturbation. A gas puff current was switched on into the edge and ramped from 0 to $2000 \mathrm{~A}$ in $1 \mathrm{~ms}$. Thereafter the gas flow was left constant and the profiles evolved towards a steady state. This time evolution is summarized in Fig. 5 and its behavior appears qualitatively consistent with observations in DIII-D.

\section{CONCLUSIONS}

The first tests that were conducted with CORSICA 2 demonstrate code robustness and ability to deal with experiment-relevant conditions. Despite the preliminary nature of this
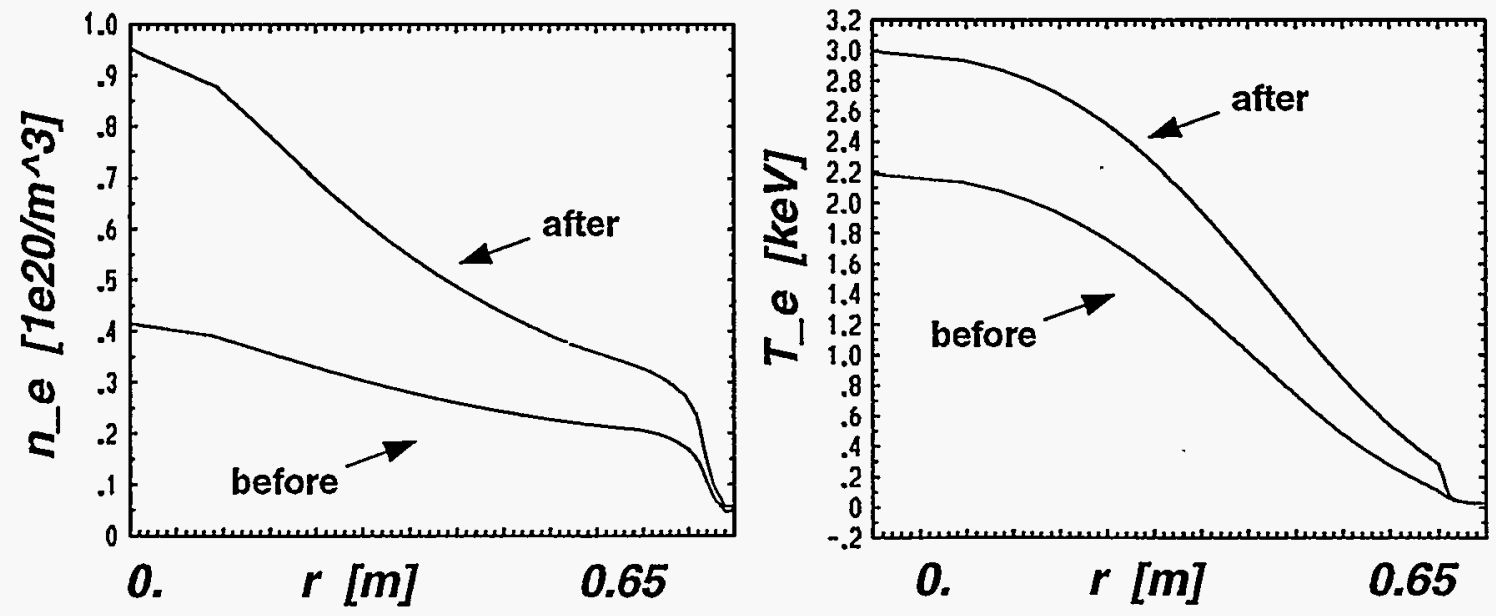

Fig. 3. L-H transition:core-edge profiles for density and electron temperature 

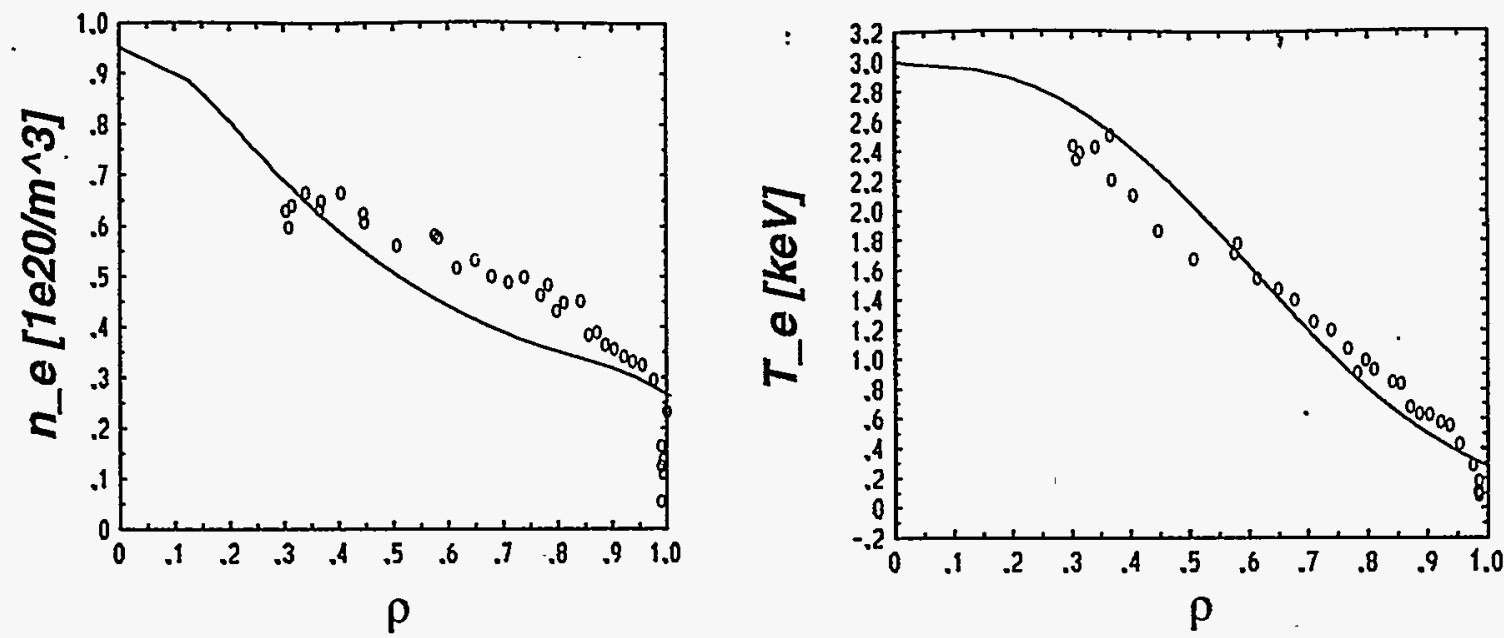

Fig. 4. L-H transition:core density and electron temperature profiles in CORSICA 2 after the transition compared to experimental data from Thomson scattering measurements (circles). $\rho$ is the normalized toroidal flux.
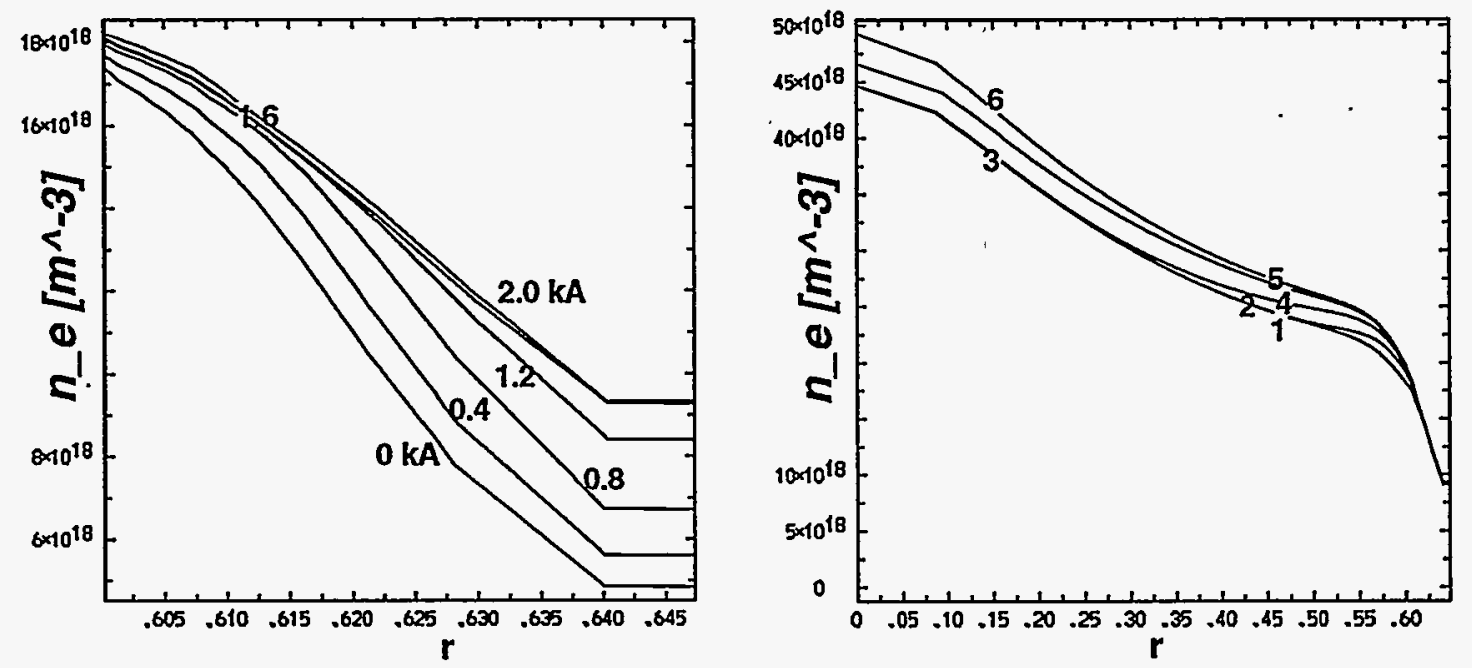

Fig. 5. Gas puffing: on the left, edge density profile vs. radial coordinate during the initial ramp of $.1 \mathrm{~ms}$ ( 0 to $2000 \mathrm{~A}$ in $400 \mathrm{~A}$ increments). On the right: core-edge density at $t=0,1 \mathrm{~ms}, 10 \mathrm{~ms}$ $100 \mathrm{~ms}, 1 \mathrm{~s}$ and $100 \mathrm{~s}$ (regime) for a constant gas flow at $2000 \mathrm{~A}$ (curves 1-6).

work, the results obtained so far are credible and in good agreement with experimental data.

Work is in progress towards both improving the algorithm performance and refining the physics models used in CORSICA 1 and UEDGE.

\section{REFERENCES}

[1] A. Tarditi et al., Bull. Am. Phys. Soc., 38 (1993), 2103.

[2] M. Balmaens et al., Proc. 22nd EPS Conf. on Plasma Phys. and Controlled Fusion, Bournemouth, U.K. (1995)

[3] A. Taroni, these proceedings

[4] J. A. Crotinger, et al., Bull. Am. Phys. Soc., 38 (1993), 2016.

[5] T. D. Rognlien, et al., Contrib. Plasma Phys., 34 (1994), 362.

[6] P. F. Dubois, Computers in Physics, 8 (1994), 70

\section{ACKNOWLEDGMENTS}

Work performed for U.S. D.O.E. at LLNL under contract W-7405-ENG-48 
. 

University of California . Livermore, California 94551

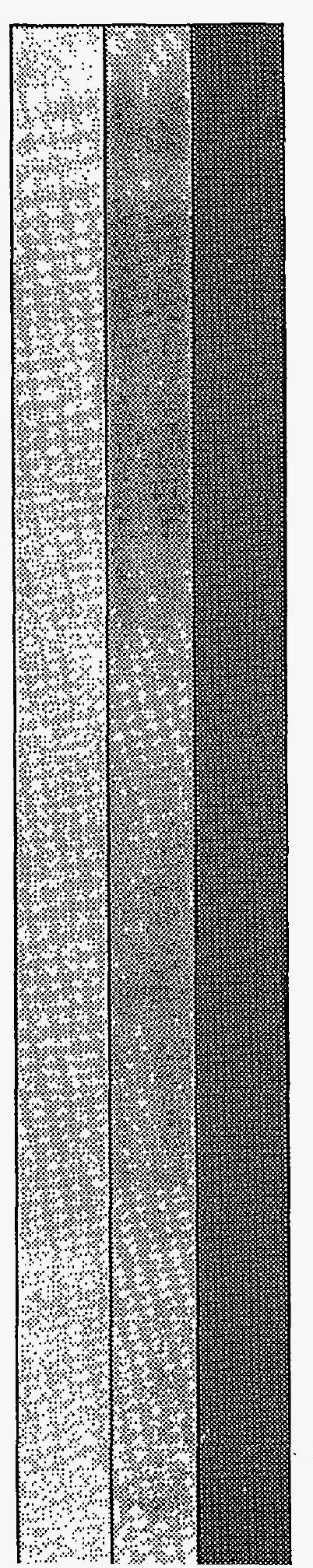

